\title{
Analysis on the Status Quo and Development Trend of Computer-aided Translation Technology
}

\author{
Yani Wang \\ Xishuangbanna Vocational and Technical College, Jinghong, Xishuangbanna, Yunnan, 666100
}

Keywords: status quo, development trend, computer-aided translation

\begin{abstract}
The flourishing development of the modern social translation industry has made computer-aided translation tools more and more important in the translation process. Computer-aided translation tools can greatly help translators, and their role in the translation process cannot be underestimated, but these translation aids also have certain deficiencies. A better understanding of the advantages and disadvantages of computer translation aids in the translation process can help translators to better utilize the advantages of translation tools in the translation process, avoid their disadvantages, and make the translation tools more prominent in the translation process.
\end{abstract}

\section{Introduction}

Currently, the Internet is gradually becoming a multilingual online world. According to statistics, from 2000 to 2005, the number of people using English on the Internet increased by 126.9\%, while the number of people using Russian increased by 664.5\%, the number of people using French increased by $235.9 \%$, and the number of people using Portuguese increased by $327.3 \%$. The number of people using Chinese has increased by 309.6\%. New information and new knowledge are increasing in the information age, and there is a situation of information explosion. According to statistics, there are currently 165,000 scientific and technical publications published in the world, and an average of 20,000 scientific papers are published every day. According to a survey of the world's translation market by international authorities, the size of the translation market in the world exceeded RMB 65 billion in 2010, and this figure is still growing at a rate of 30\% per year. In 2007, the volume of translations for web pages alone reached \$1.7 billion. Since China joined the WTO, the scale of China's translation market has exceeded 100 yuan. However, China's translation capacity is seriously inadequate. The existing translation companies in China can only digest about $10 \%$ of foreign language translations. Due to language blockage, China has lost a lot of opportunities in international competition. Traditional manual translation has obviously been difficult to meet the growing demand for translation. The need to use computer translation technology and tools is becoming more and more urgent, because it is to improve translation efficiency and solve language bottlenecks. The only way out.

\section{Advantages of computer-aided translation tools}

The biggest difference between computer-aided translation tools and machine translation tools lies in the degree of participation in human translation. Computer-aided translation is mainly based on people and tools, while machine translation is $100 \%$ translated by machine. Due to the high error rate, the role of machine translation in the translation process has gradually faded, but translation tools such as online translation that can be classified as machine translation still have a significant effect. Due to the rapid development of technology and the gradual popularization of the Internet, the use of online translation tools is becoming more and more common, especially Baidu translation and Google translation. The online translation tools used by students from both translation majors and non-translating majors, as well as professional translators. Despite the existence of a certain error rate in online translation, and sometimes the translations provided by online translation tools are flawed and can not improve any reference value. But a significant advantage of the online 
translation tool is still not negligible, which is to provide translators with translation ideas. Just like a thousand readers, there will be a thousand Hamlet, and there will be many different translations in one sentence. Although the translation provided by the online translation tool is not completely desirable, the use of words and sentence structure can provide a reference for the translator and explore the translator's ideas to find a better translation.

One of the most prominent advantages of computer-assisted translation tools is the ability to greatly improve the efficiency of translators and save a lot of time. Computer-aided translation tools can improve translation efficiency in the following ways. One is the powerful memory and termbase. In the case of Trados and Wordfast, when using both translation tools for translation, the corresponding memory and termbase can be imported at the beginning of the translation project, but both tools have their use in memory and terminology. Advantages: When using Trados Studio, the matching rate of the memory can be set. During the translation process, once the matching ratio between the original text and a segment in the memory reaches the set value, the segment will be automatically jumped out for the translator's reference. However, the term requires the translator to manually search, but it is also very convenient; when using Wordfast, the term is automatically identified without searching, but the contents of the memory need to be manually searched by the translator. Both tools have their own advantages, and they can improve translators' translation efficiency and save time with powerful memory functions and terminology. The second is to automatically make the translation completely in the original format, without spending extra time for document format processing. This is also a powerful part of computer-aided translation tools. The translation projects undertaken by the current translation companies all have a fixed format. If you use common tools (such as word) to translate and then modify the format, it will not only take a lot of time, but also it is difficult to ensure the format is completely consistent. However, the computer-aided translation tool can not only automatically segment the segments, but also make the translation process more concise and clear, and ensure that the original translation format is completely consistent, so that the translator has no worries. The third is to achieve uniformity of translation of fixed sentences. Whether it is for personal translation or teamwork translation, it is necessary to ensure the uniformity of the fixed statement translation. On the one hand, it is necessary to ensure the unification of the previous translations, on the other hand, it is also necessary to ensure the unity of multiple translators. Take Trados and Wordfast as examples, the memory of both tools can be shared. During the translation process, the translator completes the segment translation and then synchronously records the translation into the shared memory. The translator can find the shared memory at any time, unify the translation, and refer to the contents of the memory, which not only ensures the uniformity of the translation, but also does not express contradictions. It also improves translation efficiency.

When the online dictionary was not popular yet, the translation of the word can only rely on the paper-based dictionary, which is very time consuming. But like online translation tools, online dictionary tools are increasingly used by translators. Common online dictionary tools like Dao, Jinshan and Haizi can greatly reduce the translator's translation burden. In the past, when you encountered new words or uncertain words in the translation process, you could only flip through the paper dictionary. Now with the online dictionary, you can solve the problem quickly with a click of the mouse. Although the translator's vocabulary is still important, it is no longer a factor that will cause problems for the translator. Even if the paper dictionary seems to be more detailed, you can find the contents of the practical dictionary such as the 21st Century English-Chinese Dictionary and the Collins English-Chinese Dictionary, and you can also find the paper dictionary. The latest usage that is not available. Vocabulary is no longer a heavy burden for translators.

\section{Internet cloud computing translation model construction}

Cloud computing is the fourth IT industry revolution after large computers, personal computers, and the Internet. A more common explanation for cloud computing is that the "cloud" of cloud computing is the resource on the server cluster existing on the Internet. It includes hardware resources such as servers, storage, and CPU, as well as software resources such as application 
software and integrated development environment. The local computer only needs to send a demand message through the Internet. At the remote end, there are thousands of computers that provide the required resources according to the request and return the result to the local computer. In the process, the local computer hardly needs it. For any operation, all processing is done by a computer cluster provided by the cloud computing provider. With the rapid development of the cloud computing industry, it has been increasingly used in various industries. The concept of "cloud translation" is naturally proposed by the translation community. The cloud translation platform is a cloud computing application that collects, transmits, stores and utilizes a large amount of corpus information. Through this platform, the translation talents can be stored in the hundreds of thousands of people, and can serve a large number of industries in a wide range of industries. Document translation work. According to the language, type, field and translation requirements of the translation project, the most appropriate translation and reviewers can be called through the cloud translation platform to form a project team for network collaborative translation, and through machine translation and computer-assisted translation. Extensive use of technologies such as cross-language search, 24/7 updated translation corpus, providing online translation or human translation services for the public and various industries to instantly change the service model of the traditional translation industry; This platform also collects scattered translation market needs. In short, applying the cloud translation platform to the Chinese multilingual translation industry can effectively pool resources, improve translation quality and quality, and reduce individual costs through scale effects, allowing users to access them in an on-demand and easily scalable manner via the Internet. Rich, convenient and efficient service. At present, cloud electronic dictionary technology based on cloud translation platform has gradually matured and been applied. For example: Microsoft's Bing Dictionary and NetEase's well-known dictionary, which use a cloud-based architecture and cloud service model, can simultaneously search a large number of new words appearing on the Internet; can achieve word and sentence on the website, and A more accurate translation of English and Chinese translations; it is convenient for netizens to browse English websites and read $80 \%$ of web content. However, the true high-quality translation and the best performance carrier for carrying this kind of translation are far from being realized. The real meaning of the cloud translation platform needs to have a longer way to go. Fortunately, under the eviction of the Internet and cloud computing, I hope that Dawning has emerged, which requires multilingual computer-aided translation, computer-supported collaborative work, cloud computing, optical recognition, unified communications, Internet application development, and data centers continuous research and development of the technology.

\section{Conclusion}

Computer translation in China is gradually becoming a hotspot in the translation industry, but this field is a combination of information technology and translation research. It covers a wide range and is difficult to study. It is impossible to study machine translation technology in any single subject but every aspect of it.

Moreover, China's special research on the computer-aided translation industry is almost blank. This gap needs to be filled. At the same time, the study of Chinese grammar needs to be strengthened. The Chinese grammar and English grammar are far different in structure, if it is copied from Western computer translation. Theory will make the study of machine translation Chinese difficult, so the theoretical research based on Chinese grammar needs to be further strengthened. Only in this way can the bottleneck of Chinese machine translation be solved. For English majors, no matter how powerful the translation software is, these software are just an aid. They can never replace our brains, so learning English is the last word.

\section{References}

[1] Fan Yi. I and the translation software [J]. Chinese translation, 2000 (3). 
[2] Huang Junhong, Huang Ping, Fan Yun. A Review of the Parallel Corpus of Special Purpose Language Translation [J]. Journal of Chongqing University: Social Science Edition, 2004(6).

[3] Qian Duoxiu. Thinking on the Teaching of "Computer Aided Translation" Course [J]. Chinese Translation, 2009(4).

[4] Zhang Yihua. Discussion on Computer-Aided Translation [J]. Shanghai Science and Technology Translation, 2002(1).

[5] Li Bin, Tan Yueqin. On China's Machine Translation Software [J]. Journal of Sichuan College of Education, 2004(3): 52-53. 